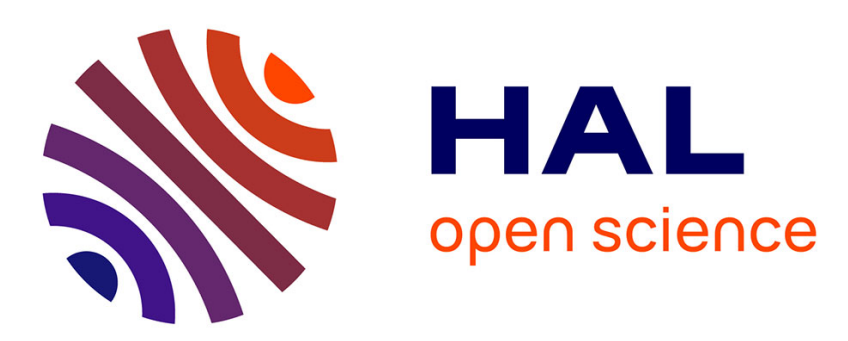

\title{
Constrained Multicriteria Sorting Method Applied to Portfolio Selection
}

\author{
Jun Zheng, Olivier Cailloux, Vincent Mousseau
}

\section{To cite this version:}

Jun Zheng, Olivier Cailloux, Vincent Mousseau. Constrained Multicriteria Sorting Method Applied to Portfolio Selection. Algorithmic Decision Theory, Oct 2011, New Brunswick, New Jersey, United States. pp.331-343, 10.1007/978-3-642-24873-3_25. hal-00951743

\section{HAL Id: hal-00951743 https://hal.science/hal-00951743}

Submitted on 25 Feb 2014

HAL is a multi-disciplinary open access archive for the deposit and dissemination of scientific research documents, whether they are published or not. The documents may come from teaching and research institutions in France or abroad, or from public or private research centers.
L'archive ouverte pluridisciplinaire HAL, est destinée au dépôt et à la diffusion de documents scientifiques de niveau recherche, publiés ou non, émanant des établissements d'enseignement et de recherche français ou étrangers, des laboratoires publics ou privés. 


\title{
Constrained Multicriteria Sorting Method Applied to Portfolio Selection
}

\author{
Jun Zheng, Olivier Cailloux, and Vincent Mousseau \\ Industrial Engineering Laboratory, Ecole Centrale Paris \\ Grande Voie des Vignes 92295 Châtenay Malabry Cedex, France \\ \{jun.zheng, olivier.cailloux, vincent.mousseau\}@ecp.fr
}

\begin{abstract}
The paper focuses on portfolio selection problems which aim at selecting a subset of alternatives considering not only the performance of the alternatives evaluated on multiple criteria, but also the performance of portfolio as a whole, on which balance over alternatives on specific attributes is required by the Decision Makers (DMs).

We propose a two-level method to handle such decision situation. First, at the individual level, the alternatives are evaluated by the sorting model ELECTRE TRI which assigns alternatives to predefined ordered categories by comparing alternatives to profiles separating the categories. The DMs' preferences on alternatives are expressed by some assignment examples they can provide, which reduces the DMs' cognitive efforts. Second, at the portfolio level, the DMs' preferences express requirements on the composition of portfolio and are modeled as constraints on category size. The method proceeds through the resolution of a Mixed Integer Program (MIP) and selects a satisfactory portfolio as close as possible to the DMs' preference.

The usefulness of the proposed method is illustrated by an example which integrates a sorting model with assignment examples and constraints on the portfolio definition. The method can be used widely in portfolio selection situation where the decision should be made taking into account the performances of individual alternatives and portfolio simultaneously.
\end{abstract}

Keywords: Multicriteria decision aiding; Portfolio selection; Preference elicitation

\section{Introduction}

Let us consider the student enrollment in universities every year. Universities want to select students with good performances on several criteria (such as GPAs, motivation, maturity, ... ). At the same time, the selected students should satisfy some specific requirements at a collective level. For instance, the number of students in each department should be more or less balanced. Each department tries to achieve a gender (nationality, etc.) diversity. Moreover, the positions available are limited. Therefore, the universities face a decision which consists of selecting a certain number of students, designing a waiting list and rejecting the other students (see similar example in universities [7, other examples are 
available in the book 28]). Another example of such portfolio selection problems concerns allocating grants to research proposals. The committee evaluates the merit of the proposal, including originality, novelty, rigor and the ability of the researchers to carry out the research individually. On a whole level, they try to balance the funding among disciplines, institutions and even regions. Therefore, a decision is to be made to select certain research proposals within limited budget.

The two problems above share some characteristics. Firstly, they involve evaluating individual alternatives according to their performances on multiple criteria. Secondly, a portfolio is to be selected based not only on individual alternative's performance, but also on the performance of the whole portfolio. Such situation typically corresponds to a portfolio selection problem.

There is a large number of methods in literature for evaluating and selecting portfolios [15|25|16|18]. Cost-benefit analysis [24], multiattribute utility theory [13], weighted scoring [9] are widely used. Some researchers combine preference programming with portfolio selection considering incomplete preference information [17/18. However, to our knowledge, Multiple Criteria Decision Aiding (MCDA) outranking methods have not been applied to portfolio selection problem. Furthermore, the ability of the methods to express sophisticated preference on portfolios has little been explored. A balance model [14] is developed which measures the distribution of specific attributes by dispersion and uses such measurement to select subsets of multiattribute items. 15. uses constraints to eliminate the ones which do not fit in the requirement on whole portfolio.

We propose a two-level method for such portfolio selection problems. At individual level, the paper uses ElECTRE TRI method [2627] to evaluate the alternatives on multiple criteria, which assigns alternatives to predefined ordered categories by comparing an alternative with several profiles. The DMs' preference on individual evaluation can be taken into account by some assignment examples. At portfolio level, a wide class of preferences on portfolios (resource limitation, balance of the selected items over an attribute...) are represented using general category size constraints. An optimization procedure is performed by solving a MIP to infer the values of preference parameters and to identify a satisfactory portfolio.

The paper is organized as follows. Section 2 formulates portfolio selection problem as a constrained multicriteria sorting problems. Section 3 presents a mathematical program which computes the portfolio that best matches the DMs' preferences. Section 4 illustrates the proposed method with an example. The last section groups conclusions.

\section{Problem Formulation}

\subsection{Evaluating Alternatives with Electre Tri Method}

Alternatives to be included on a portfolio are evaluated by an outranking method ELECTRE TRI 26]27. This method assigns alternatives to predefined ordered categories by comparing the alternatives to profiles which define the frontiers 
of two successive categories. For example, for enrollment problem described in Section 1, the DMs want to sort the students into three categories: accepted, waiting list or rejected according to students' performances on multiple criteria. Thus the two profiles could be two frontiers which separate these three categories.

Formally, ELECTRE TRI assigns each alternative of a set $A=\left\{a_{1}, a_{2}, \ldots, a_{n}\right\}$ to $k$ pre-defined ordered categories $\mathrm{Cat}_{1} \ll \mathrm{Cat}_{2} \ll \ldots \ll \mathrm{Cat}_{k}$. $K$ denotes the set of indices of the $k$ categories $(K=\{1,2, \ldots, k\})$. The alternatives are evaluated on $m$ criteria. Let $J$ denote the set of the indices of the criteria $g_{1}, g_{2}, \ldots, g_{m}$ $(J=\{1,2, \ldots, m\})$. For all $j \in J, a \in A, g_{j}(a)$ represents the evaluation of $a$ with respect to the $j$ th criteria. In what follows we assume, without loss of generality, that preference increases with the value on each criterion. $b_{h}$ is the upper limit of category $h$ and the lower limit of category $h+1, h=1,2, \ldots, k-1$. In other words, the frontier separating two categories is represented by the evaluations on the set of criteria of the profile $b_{h}: g_{j}\left(b_{h}\right), \forall j \in J$. The assignment of an alternative $a$ results from the comparison of $a$ to the profiles $b_{1}, b_{2}, \ldots, b_{k-1}$.

ELECTRE TRI uses an outranking relation $\succcurlyeq$ which represents assertions $a \succcurlyeq b_{h}$ whose meaning is " $a$ is at least as good as $b_{h}$ ". In order to validate the assertion $a \succcurlyeq b_{h}$, a "sufficient" majority of criteria should be in favor of this assertion. A set of weights coefficients $\left(w_{1}, w_{2}, \ldots, w_{m}\right)$ which sum to 1 representing relative importance of the criteria is additively used in the concordance test when computing the strength of the coalitions of criteria being in favor of the assertion $a \succcurlyeq b_{h}$.

EleCtre TRI builds a concordance index $C\left(a, b_{h}\right) \in[0,1], \forall a \in A, \forall h \in K$, defined as $C\left(a, b_{h}\right)=\sum_{j \in J \mid g_{j}(a) \geq g_{j}\left(b_{h}\right)} w_{j}$. It represents the degree of credibility of the assertion $a \succcurlyeq b_{h}$. The assertion $a \succcurlyeq b_{h}$ is considered to be valid if $C\left(a, b_{h}\right) \geq \lambda, \lambda$ being the "majority level" such that $\lambda \in[0.5,1]$.

We consider a simplified ELECTRE TRI method which ignores discrimination thresholds (preference and indifference threshold) and veto threshold involved in the standard non-discordance condition 23. Such simplification is in line with the axiomatic study of Bouyssou and Marchant [2]3].

Given the outranking relation $\succcurlyeq$, alternatives are assigned to categories on the basis of the way they compare to profiles $b_{h}$. ELECTRE TRI proposes two assignment procedures (so-called pessimistic and optimistic rule). In this paper we consider only the pessimistic rule which assigns alternative $a$ to the highest category Cat $\mathrm{C}_{h}$ for which $a \succcurlyeq b_{h-1}$ and not $a \succcurlyeq b_{h}$.

So as to implement EleCtRE TRI, an elicitation process is necessary to determine the values of preference parameters (profiles $b_{h}$, weights $w_{j}$ and majority level $\lambda$ ). In a portfolio selection perspective, we consider DMs' preference at two levels. At alternative level, the DMs express preferences on alternatives individually. At a portfolio level, they express preferences on portfolios as a whole (resource limitation, balance of the selected items over an attribute, ...). These two preference levels are distinguished, as they are elicited in different ways, and could be provided by different DMs who have expertise and understanding of the portfolio selection at different levels. 


\subsection{DMs' Preference on Alternatives}

The DMs have little understanding of the precise semantics of the preference parameters involved in ELECTRE TRI. On the contrary, they can easily express their expertise on which category an alternative should be assigned to. Therefore, we propose to elicit the DMs' preference in an indirect way, in accordance with the disaggregation-aggregation paradigm. Instead of providing precise values for the parameters, the DMs provide assignment examples, i.e. alternatives which they are able to assign confidently to a category. For instance, in a student selection problem, the DMs may state that one particular student should be assigned to the best category (the set of accepted students). Inference procedure can thus be used to compute values for the preference parameters that best match the assignment examples. Several authors have proposed disaggregation methodologies from assignment examples expressed by the DMs. Mousseau and Słowiński use non-linear programming to infer all the parameters simultaneously 22], and some suggest to infer weights only assuming the profiles are fixed 21]. Researchers also proposed to compute robust assignment categories to which an alternative is possible to be assigned, considering all combinations of values compatible with the DMs' preference statements [1] and developed corresponding software [10. Recently, an evolutionary approach has been presented to infer all parameters of ELECTRE TRI model [12. In this paper, we assume all the preference parameters are variables and infer them by solving a MIP.

\subsection{DMs' Preference Information on Portfolios}

The DMs' preferences can also be expressed at the portfolio level (resource limitation, balance on the composition of categories w.r.t. an attribute, ...). We formalize such preferences as general constraints on category size. For example, in the student enrollment case, let us denote the category of rejected students $\mathrm{Cat}_{1}$, the category of waiting list $\mathrm{Cat}_{2}$ and the category of admitted students $\mathrm{Cat}_{3}$. Suppose the university only have 100 positions available, and such constraint can be modeled as the number of students in Cat ${ }_{1}$ cannot exceed 100. Moreover, balancing gender in the selected students (100 students in total) can also be modeled as a constraint that the number of female students in Cat ${ }_{1}$ should not be lower than 30 . Adding such constraints to the selection process may result in rejecting some male students whose performances are better than those of the accepted female students. However, such portfolio is more satisfactory for the DMs in terms of gender balance. Modeling the DMs' preference as constraints eliminates some portfolios which don't satisfy their requirements on the whole portfolio.

\section{Mathematical Program Formulation}

\subsection{Stating the Problem and Decision Variables}

Given a set of alternatives $A$, a set of criteria indices $J$, evaluations of the alternatives $g_{j}(a), \forall a \in A, j \in J$, a set of category indices $K=\{1,2, \ldots, k\}$, a 
set of profiles $b_{h}, 1 \leq h \leq k-1$, the goal of the program is to determine the performances of profiles $g_{j}\left(b_{h}\right), \forall j \in J, 1 \leq h \leq k-1$, weights $w_{j}$ and majority threshold $\lambda$, satisfying all the constraints given by the DMs in the form of assignment examples and portfolio constraints. The MIP also defines additional variables involved in the way ELECTRE TRI assigns alternatives to categories. The binary variables $C_{j}\left(a, b_{h}\right), \forall a \in A, j \in J, 1 \leq h \leq k-1$ represent the partial concordance indices such that $C_{j}\left(a, b_{h}\right)=1$ if and only if the performance of the alternative $a$ on the criterion $j$ is at least as good as the performance of the profile $b_{h}$. The continuous variables $\sigma_{j}\left(a, b_{h}\right)$ represent the weighted partial concordance indices, they are such that $\sigma_{j}\left(a, b_{h}\right)=w_{j}$ if and only if $C_{j}\left(a, b_{h}\right)=1$. Finally, binary variables $n(a, h), \forall a \in A, h \in K$ are defined so that $n(a, h)=1$ if and only if alternative $a$ is assigned to category $h$. A slack variable $s$ is used in the objective function which appreciates the ability of the ElECTRE TRI model to reproduce the assignment examples in a robust way.

The constraint $\sum_{j \in J} w_{j}=1$ is posed, and the following constraints are used to ensure a correct ordering of the profiles defining the categories: $\forall j \in J, 2 \leq$ $h \leq k-1: g_{j}\left(b_{h-1}\right) \leq g_{j}\left(b_{h}\right)$.

\subsection{Constraints Stemming from Preferences at Individual Level}

The set of assignment examples $E$ is the set of pairs $(a, h) \in A \times K$ specifying that alternative $a$ is assigned to $\mathrm{Cat}_{h}$. Recall that satisfying an assignment example $(a, h)$ amounts to satisfy both $\sum_{j \in J: g_{j}(a) \geq g_{j}\left(b_{h-1}\right)} w_{j} \geq \lambda$ and $\sum_{j \in J: g_{j}(a) \geq g_{j}\left(b_{h}\right)} w_{j}<\lambda$.

The sum of support in favor of the outranking of an alternative $a$ over a profile $b_{h}, \sum_{j \in J: g_{j}(a) \geq g_{j}\left(b_{h}\right)} w_{j}$, can also be written $\sum_{j \in J} C_{j}\left(a, b_{h}\right) w_{j}$ with $C_{j}\left(a, b_{h}\right)$ equal to one iff $g_{j}(a) \geq g_{j}\left(b_{h}\right)$. Constraints (11) define the binary variables $C_{j}\left(a, b_{h}\right), \forall j \in J, a \in A, 1 \leq h \leq k-1$, where $\varepsilon$ is an arbitrary small positive value, and $M$ is an arbitrary large value. See also Fig. 1

$$
\frac{1}{M}\left(\left(g_{j}(a)-g_{j}\left(b_{h}\right)\right)+\varepsilon\right) \leq C_{j}\left(a, b_{h}\right) \leq \frac{1}{M}\left(g_{j}(a)-g_{j}\left(b_{h}\right)\right)+1 .
$$

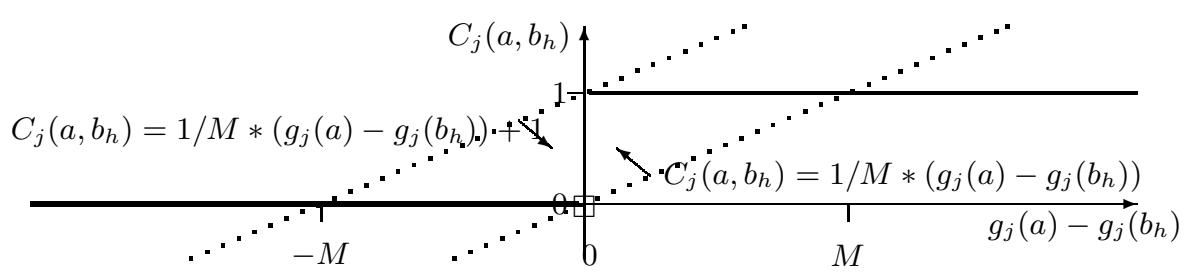

Fig. 1. constraining $C_{j}\left(a, b_{h}\right)$ to the appropriate value

The following constraints define the variables $\sigma_{j}\left(a, b_{h}\right)$ representing the sum of the support in favor of the assertion " $a$ is at least as good as $b_{h}$ " while avoiding 
the non-linear expression $\sigma_{j}\left(a, b_{h}\right)=C_{j}\left(a, b_{h}\right) w_{j}$ [19. See also Fig. [2.

$$
\forall j \in J, a \in A, 1 \leq h \leq k-1:\left\{\begin{array}{l}
\sigma_{j}\left(a, b_{h}\right) \leq w_{j} \\
\sigma_{j}\left(a, b_{h}\right) \geq 0 \\
\sigma_{j}\left(a, b_{h}\right) \leq C_{j}\left(a, b_{h}\right) \\
\sigma_{j}\left(a, b_{h}\right) \geq C_{j}\left(a, b_{h}\right)+w_{j}-1 .
\end{array}\right.
$$

We also define, for simplicity of use in the next constraints, $\forall j \in J, a \in A$ : $\sigma_{j}\left(a, b_{0}\right)=w_{j}$ and $\sigma_{j}\left(a, b_{k}\right)=0$.

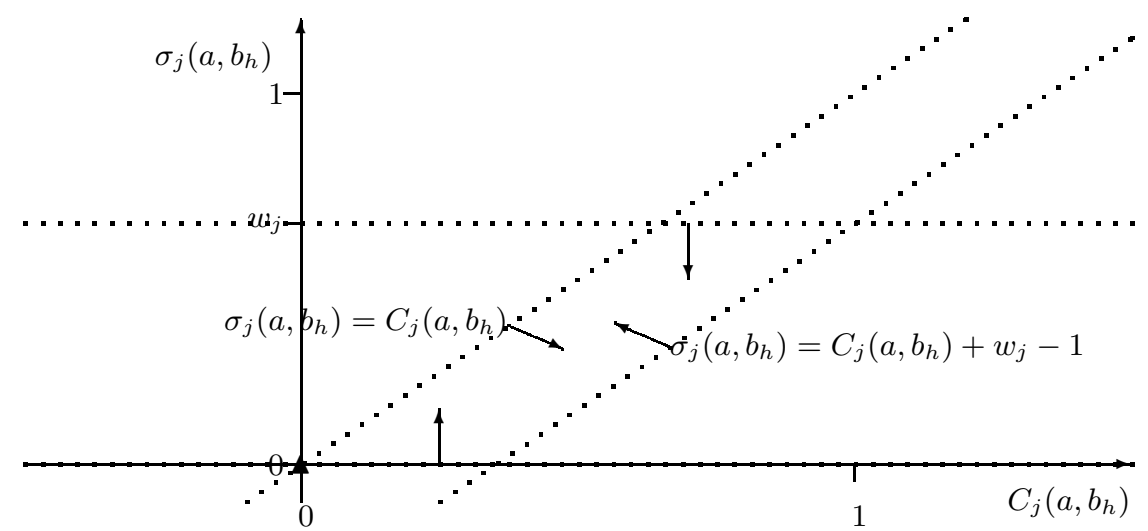

Fig. 2. constraining $\sigma_{j}\left(a, b_{h}\right)$ to the appropriate value

Finally, we need to ensure that each assignment example is assigned to the class specified by the DMs. The variable $s$ is a slack variable used in the objective function.

$$
\forall(a, h) \in E: \sum_{j \in J} \sigma_{j}\left(a, b_{h}\right)+s+\varepsilon \leq \lambda \leq \sum_{j \in J} \sigma_{j}\left(a, b_{h-1}\right)-s .
$$

\subsection{Constraints Stemming from Preferences at Portfolio Level}

Suppose the DMs want to impose, in a student selection problem, that at least 30 students in the best category (i.e. $\mathrm{Cat}_{k}$ ) are females. To model this, we define a function Gender on the set of alternatives that equals one if the student $a$ is a female student and zero otherwise, and set as a constraint that the sum of $\operatorname{Gender}(a)$ on each alternative $a$ assigned to $\mathrm{Cat}_{k}$ should be at least 30 $\left(\sum_{a \rightarrow \mathrm{Cat}_{k}} \operatorname{Gender}(a) \geqq 30\right)$. In a project selection problem, suppose the DMs want to make sure that the sum of the costs of the selected projects (say, the projects in the best category) do not exceed the available budget $x$. A function Cost would be defined on the set of alternatives representing their cost 
attribute, and a constraint is added to ensure that the sum of $\operatorname{Cost}(a)$ on alternatives $a$ assigned to the best category should be no greater than the budget $\left(\sum_{a \rightarrow \mathrm{Cat}_{k}} \operatorname{Cost}(a) \leq x\right)$.

More generally, portfolio preferences are represented as a set $N$ of tuples $\left\langle h, \underline{n_{h}}, \overline{n_{h}}, P\right\rangle, 1 \leq h \leq k, \underline{n_{h}}, \overline{n_{h}} \in \mathbb{R}, P$ a function from $A$ to $\mathbb{R}$, representing the constraint that the preferential model inferred by the program should be such that the number of alternatives from $A$ assigned to $\mathrm{Cat}_{h}$ weighted by their attribute $P$ should be at least $\underline{n_{h}}$ and at most $\overline{n_{h}}: \underline{n_{h}} \leq \sum_{a \rightarrow \text { Cat }_{h}} P(a) \leq \overline{n_{h}}$.

The following constraints define the binary variables $n(a, h), \forall a \in A, 1 \leq$ $h \leq k$, so that $n(a, h)$ equals one iff $a$ is assigned to category Cat ${ }_{h}$, that is, $\sum_{j \in J} \sigma_{j}\left(a, b_{h-1}\right) \geq \lambda$ and $\sum_{j \in J} \sigma_{j}\left(a, b_{h}\right)<\lambda$. The first constraints force that $n(a, h)=1$ requires that $a$ goes to category $h$, and the last ones force that exactly one $n(a, h)$ among all $h$ equals one.

$$
\begin{gathered}
\forall a \in A, 1 \leq h \leq k:\left\{\begin{array}{c}
n(a, h) \leq 1+\sum_{j \in J} \sigma_{j}\left(a, b_{h-1}\right)-\lambda, \\
n(a, h) \leq 1+\lambda-\sum_{j \in J} \sigma_{j}\left(a, b_{h}\right)-\varepsilon .
\end{array}\right. \\
\forall a \in A: \sum_{1 \leq h \leq k} n(a, h)=1 .
\end{gathered}
$$

These variables permit to guarantee the desired group sizes.

$$
\forall\left\langle h, \underline{n_{h}}, \overline{n_{h}}, P\right\rangle \in N: \underline{n_{h}} \leq \sum_{a \in A} n(a, h) P(a) \leq \overline{n_{h}} .
$$

\subsection{Objective Function and Resolution Issues}

In order to maximize the separation between the sum of support and the majority threshold, the objective of the MIP is set to maximize the slack variable $s$ as defined in Constraints (3). The slack variable evaluates the ability of the ELECTRE TRI model to "reproduce" the assignment examples in a robust way.

However the preference information of the DMs does not lead univocally to a single compatible portfolio. The optimization procedure finds out one of the compatible portfolios. In an interactive perspective, the DMs can provide further preference information considering the results of the MIP, and the information can be added to the optimization procedure to get a more satisfactory portfolio. The decision aiding process can proceed with several interactions until the DMs are content with the selected portfolio.

\section{Illustrative Example}

Let us illustrate the method with the following hypothetical decision situation. A government board has the responsibility to choose which research projects to finance among a list of 100 research proposals. The selection process involves 
sorting these proposals into three categories: projects that are considered very good and should be funded (category Good); projects that are good and should be funded if supplementary budget can be found (category Average); projects that are of insufficient quality and should not be funded (category Bad). To sort these projects in these three categories, the board agrees to use the following six criteria.

sq The project's scientific quality, evaluated on a 5 points ordinal scale.

rq The proposal's writing quality, evaluated on a 5 points ordinal scale.

a The proposal's adequacy with respect to the government priorities, evaluated on a 3 points ordinal scale.

te The experience of the researcher teams submitting the project, evaluated on a 5 points ordinal scale.

ic Whether the proposal includes international collaboration, a binary assessment.

ps The researchers' publication score evaluated by an aggregate measure of the total quality of publications of the researchers involved in the proposal (evaluated on a $[0,100]$ scale).

The scales on all criteria are defined such that a greater value corresponds to a better evaluation.

Supplementary to these six criteria, the 100 projects to be evaluated are described by three attributes: the research domain to which the project belongs (Operational Research (OR), Artificial Intelligence (AI) or Statistics); the budget the project asks funding for; the originating country. Table 1 shows the data about the first 7 projects in the list (complete data lists for the whole example are available at http://www.lgi.ecp.fr/ mousseau/ADT2011/). In order to determine an appropriate preference model, the board gives as a first stage 30 examples of past research proposals whose performances on the six criteria and final quality evaluation are known. A part of this data is shown in Table 2 .

Table 1. Some of the research projects to be evaluated. The budget is in tens of $\mathrm{E}$.

\begin{tabular}{lrrrrrrllll}
\hline & \multicolumn{4}{c}{ evaluations criteria } & \multicolumn{4}{c}{ descriptive attributes } \\
\hline Project & rq & ps & a & sq & te & ic & \multicolumn{3}{c}{ budget domain } & country \\
\hline Pr001 & 2 & 47 & 2 & 3 & 1 & 0 & 27 & Stat. & Germany \\
Pr002 & 2 & 3 & 2 & 4 & 4 & 0 & 29 & Stat. & France \\
Pr003 & 5 & 63 & 1 & 5 & 1 & 0 & 20 & Stat. & Italy \\
Pr004 & 1 & 92 & 3 & 5 & 5 & 1 & 34 & AI & Germany \\
Pr005 & 4 & 13 & 2 & 4 & 2 & 0 & 32 & Stat. & Germany \\
Pr006 & 5 & 5 & 3 & 5 & 1 & 0 & 22 & Stat. & Netherlands \\
Pr007 & 1 & 27 & 3 & 2 & 5 & 1 & 34 & OR & Germany \\
$\vdots$ & & & & & & & & & \\
\hline
\end{tabular}


Table 2. Some research project examples and their respective assignments.

\begin{tabular}{lrrrrrll}
\hline Project & rq & ps & a & sq & te & ic & Cat \\
\hline Ex01 & 4 & 50 & 2 & 3 & 3 & 0 & Average \\
Ex02 & 4 & 85 & 3 & 1 & 5 & 1 & Good \\
Ex03 & 3 & 95 & 1 & 2 & 5 & 1 & Average \\
Ex04 & 5 & 91 & 2 & 2 & 5 & 1 & Good \\
Ex05 & 5 & 89 & 1 & 5 & 3 & 0 & Good \\
Ex06 & 3 & 5 & 3 & 2 & 2 & 1 & Average \\
$\vdots$ & & & & & & & \\
\hline
\end{tabular}

The inference program is run with these assignment examples, and without supplementary portfolio constraints. Table 3 lists the resulting profiles and weights. Note that the profiles performances values in all our tables have been rounded up. Because each alternative used in this example has integer performance values on all criteria, doing so does not impact the way each alternative compares to these profiles. The resulting preference model is used to evaluate the 100 research projects, which leads to 22 projects being evaluated as good projects. The board is not satisfied with this set of projects because accepting these projects induces a total funding cost of 718 which exceeds the available budget (400). The program is thus run again with a supplementary constraint on the sum of the budget of the projects being assigned to the Good category to ensure that it stays below the available budget.

Table 3. Profiles, weights and majority threshold inferred during the first stage.

\begin{tabular}{rrrrrrrr}
\hline & rq & ps & a & sq & te & ic & $\lambda$ \\
\hline b1 & 2 & 73 & 4 & 1 & 2 & 1 & \\
$\mathrm{~b} 2$ & 4 & 96 & 4 & 5 & 3 & 1 & \\
\hline \hline $\mathrm{w}$ & 0.2 & 0.2 & 0 & 0.2 & 0.2 & 0.2 & 0.5 \\
\hline
\end{tabular}

This second stage inference yields other profiles and weights, given in Table 4. and a new list of assignments of which a part is displayed in Table 5. At this stage 11 projects are assigned to category Good and therefore are to be financed, leading to a total cost below 400. However the board is not fully satisfied yet because one domain is largely favored by this result, as the AI domain has 7 projects selected whereas only 1 project in the OR domain is to be financed. In a third stage, the inference program is thus run again with a new constraint requiring that the domain OR has at least 2 projects in the category Good. The final assignment results, shown partly in Table 6] are considered satisfactory. 
Table 4. Profiles, weights and majority threshold inferred with supplementary budget constraint.

\begin{tabular}{lrrrrrrr}
\hline & $\mathrm{rq}$ & $\mathrm{ps}$ & $\mathrm{a}$ & $\mathrm{sq}$ & te & ic & $\lambda$ \\
\hline $\mathrm{b} 1$ & 2 & 2 & 2 & 1 & 2 & 1 & \\
$\mathrm{~b} 2$ & 3 & 84 & 2 & 4 & 3 & 2 & \\
\hline \hline $\mathrm{w}$ & 0.143 & 0.143 & 0.143 & 0.143 & 0.286 & 0.143 & 0.643 \\
\hline
\end{tabular}

Table 5. A part of the assignment of the research projects with the preference model inferred during the second stage.

\begin{tabular}{|c|c|c|c|c|c|c|c|c|c|c|}
\hline Project & $\mathrm{rq}$ & ps & $\mathrm{a}$ & sq & te $\mathrm{i}$ & ic & budget & domain & country & Cat \\
\hline Pr001 & 2 & 47 & 2 & 3 & 1 & 0 & 27 & Stat. & Germany & $\mathrm{Bad}$ \\
\hline Pr002 & 2 & 3 & 2 & 4 & 4 & 0 & 29 & Stat. & France & Average \\
\hline Pr003 & 5 & 63 & 1 & 5 & 1 & 0 & 20 & Stat. & Italy & Bad \\
\hline Pr004 & 1 & 92 & 3 & 5 & 5 & 1 & 34 & $\mathrm{AI}$ & Germany & Good \\
\hline Pr005 & 4 & 13 & 2 & 4 & 2 & 0 & 32 & Stat. & Germany & Average \\
\hline Pr006 & 5 & 5 & 3 & 5 & 1 & 0 & 22 & Stat. & Netherlands & Bad \\
\hline Pr007 & 1 & 27 & 3 & 2 & 5 & 1 & 34 & OR & Germany & Average \\
\hline
\end{tabular}

The process could have continued had the board wished a better balance among the originating countries, or had they wished to consider more closely also the Average category. In case an infeasible problem had been reached at some point during the process, some constraints would have had to be relaxed or deleted. The reader will find in Mousseau et al. 20] algorithms on how to proceed for constraints relaxation.

The proposed approach has been implemented as a free, open source Java library [5]. The mathematical programs implementation relies on the JLP software package, a free and open source Java Linear Programming wrapper on top of CPLEX (the solver we used) and other commercial and free solvers. Solving the problems used in this illustrative example takes less than one minute. A study of the solving time of a related problem is available for the interested reader 4]. That study does not take into account the portfolio constraints and thus examines a simpler problem. It shows that small to medium size problems (consisting of less than eight criteria, of three categories and of less than one hundred alternatives) are solvable within ninety minutes, which is a reasonable time provided that this kind of approach is primarily used in an off-line mode. Analysis of the solving time of the problem studied here, thus with the added portfolio constraints, is left as a future work. 
Table 6. A part of the assignment of the research projects with the preference model inferred during the third stage.

\begin{tabular}{|c|c|c|c|c|c|c|c|c|c|c|}
\hline Project & rq & ps & $\mathrm{a}$ & sq & te & ic & budget & domain & country & Cat \\
\hline Pr001 & 2 & 47 & 2 & 3 & 1 & 0 & 27 & Stat. & Germany & Average \\
\hline Pr002 & 2 & 3 & 2 & 4 & 4 & 0 & 29 & Stat. & France & Average \\
\hline Pr003 & 5 & 63 & 1 & 5 & 1 & 0 & 20 & Stat. & Italy & Bad \\
\hline Pr004 & 1 & 92 & 3 & 5 & 5 & 1 & 34 & AI & Germany & Good \\
\hline Pr005 & 4 & 13 & 2 & 4 & 2 & 0 & 32 & Stat. & Germany & Average \\
\hline Pr006 & 5 & 5 & 3 & 5 & 1 & 0 & 22 & Stat. & Netherlands & Average \\
\hline Pr007 & 1 & 27 & 3 & 2 & 5 & 1 & 34 & OR & Germany & Average \\
\hline
\end{tabular}

\section{Conclusion}

The method applies constrained ELECTRE TRI model to portfolio selection problems in order to select a satisfactory portfolio considering DMs' preferences both at individual and portfolio level. Using a sorting model, the alternatives are evaluated by their intrinsic performances on criteria. Unsatisfactory portfolios which do not meet the DMs' requirements on portfolios as a whole are screened out by adding category size constraints to ElECTRE TRI model. Because of such category size constraints, the assignment of an alternative is dependent on its evaluation but also on other alternatives.

Our formalization permits to tackle the challenges the DMs may face during the decision of portfolio selection. (1) At individual level, an alternative is evaluated on multiple criteria which can be qualitative or quantitative criteria. Moreover, the DMs easily express their preferences on alternatives by assignment examples. (2) At portfolio level, the best alternatives do not necessarily compose the best portfolio. Our method takes into account the overall portfolio performance by modeling the DMs' preference on portfolio as constraints. (3) The preference information at the two levels (individual classification of alternatives and preference at the portfolio level) can be elicited from different stakeholders. (4) The proposed method involves the DMs deeply by asking them preference in an intuitive way.

The proposed method can be widely used in portfolio selection situations where the decision should be made taking into account the individual alternative and portfolio performance simultaneously. The proposed syntax of category size constraints has a broad descriptive ability for portfolio decision modeling. The method can be extended by providing robust recommendation to the DMs as a result of incomplete preference information. Moreover, the preference on portfolio level can be modeled as objectives rather than constraints of the optimization procedure, which would lead to a multiobjective problem. 


\section{References}

1. Archer, N.P., Ghasemzadeh, F.: An integrated framework for project portfolio selection. International Journal of Project Management 17(4), 207-216 (Aug 1999)

2. Bouyssou, D., Marchant, T.: An axiomatic approach to noncompensatory sorting methods in MCDM, I: The case of two categories. European Journal of Operational Research 178(1), 217-245 (Apr 2007)

3. Bouyssou, D., Marchant, T.: An axiomatic approach to noncompensatory sorting methods in MCDM, II: more than two categories. European Journal of Operational Research 178(1), 246-276 (Apr 2007)

4. Cailloux, O., Meyer, P., Mousseau, V.: Eliciting ELECTRE TRI category limits for a group of decision makers. Tech. Rep. 201109, Laboratoire Génie Industriel, Ecole Centrale Paris (Jun 2011), http://www.lgi.ecp.fr/Biblio/PDF/CR-LGI-2011-09.pdf, cahiers de recherche 2011-09

5. Cailloux, O.: ELECTRE and PROMETHEE MCDA methods as reusable software components. In: Proceedings of the 25th Mini-EURO Conference on Uncertainty and Robustness in Planning and Decision Making (URPDM 2010). University of Coimbra, Portugal, Coimbra, Portugal (Apr 2010)

6. Cailloux, O.: J-MCDA: free/libre java libraries for MCDA. http://sourceforge.net/projects/j-mcda/ (2011)

7. Cardinal, J.L., Mousseau, V., Zheng, J.: Multiple criteria sorting: An application to student selection. In: Salo, A., Keisler, J., Morton, A. (eds.) Portfolio Decision Analysis. Springer-Verlag New York Inc. (Jul 2011)

8. Chien, C.: A portfolio-evaluation framework for selecting R\&D projects. R and D Management 32(4), 359-368 (Sep 2002)

9. Coldrick, S., Longhurst, P., Ivey, P., Hannis, J.: An R\&D options selection model for investment decisions. Technovation 25(3), 185-193 (Mar 2005)

10. Dias, L., Mousseau, V.: IRIS: a DSS for multiple criteria sorting problems. Journal of Multi-Criteria Decision Analysis 12, 285-298 (2003)

11. Dias, L., Mousseau, V., Figueira, J., Clímaco, J.: An aggregation/disaggregation approach to obtain robust conclusions with ELECTRE TRI. European Journal of Operational Research 138(2), 332-348 (Apr 2002)

12. Doumpos, M., Marinakis, Y., Marinaki, M., Zopounidis, C.: An evolutionary approach to construction of outranking models for multicriteria classification: The case of the ELECTRE TRI method. European Journal of Operational Research 199(2), 496-505 (Dec 2009)

13. Duarte, B.P., Reis, A.: Developing a projects evaluation system based on multiple attribute value theory. Computers \& Operations Research 33(5), 1488-1504 (May 2006)

14. Farquhar, P.H., Rao, V.R.: A balance model for evaluating subsets of multiattributed items. Management Science 22(5), 528-539 (1976)

15. Golabi, K., Kirkwood, C.W., Sicherman, A.: Selecting a portfolio of solar energy projects using multiattribute preference theory. Management Science 27(2), 174189 (1981)

16. Hall, N.G., Hershey, J.C., Kessler, L.G., Stotts, R.C.: A model for making project funding decisions at the national cancer institute. Operations Research 40(6), 10401052 (1992)

17. Liesiö, J., Mild, P., Salo, A.: Preference programming for robust portfolio modeling and project selection. European Journal of Operational Research 181(3), $1488-$ 1505 (Sep 2007) 
18. Liesiö, J., Mild, P., Salo, A.: Robust portfolio modeling with incomplete cost information and project interdependencies. European Journal of Operational Research 190(3), 679-695 (Nov 2008)

19. Meyer, P., Marichal, J., Bisdorff, R.: Disaggregation of bipolar-valued outranking relations. In: Le Thi, H.A., Bouvry, P., Pham Dinh, T. (eds.) Proc. of MCO'08 conference. pp. 204-213. Springer, Metz, France (Sep 2008)

20. Mousseau, V., Dias, L., Figueira, J.: Dealing with inconsistent judgments in multiple criteria sorting models. 4OR 4(3), 145-158 (2006)

21. Mousseau, V., Figueira, J., Naux, J.: Using assignment examples to infer weights for ELECTRE TRI method: Some experimental results. European Journal of Operational Research 130(2), 263-275 (Apr 2001)

22. Mousseau, V., Słowiński, R.: Inferring an ELECTRE TRI model from assignment examples. Journal of Global Optimization 12(2), 157-174 (1998)

23. Mousseau, V., Słowiński, R., Zielniewicz, P.: A user-oriented implementation of the ELECTRE TRI method integrating preference elicitation support. Computers \& Operations Research 27(7-8), 757-777 (2000)

24. Phillips, L.D., Bana e Costa, C.A.: Transparent prioritisation, budgeting and resource allocation with multi-criteria decision analysis and decision conferencing. Annals of Operations Research 154(1), 51-68 (Oct 2007)

25. Rao, V.R., Mahajan, V., Varaiya, N.P.: A balance model for evaluating firms for acquisition. Management Science 37(3), 331-349 (1991)

26. Roy, B.: The outranking approach and the foundations of ELECTRE methods. Theory and Decision 31, 49-73 (1991)

27. Roy, B.: Multicriteria Methodology for Decision Aiding. Kluwer Academic, Dordrecht (1996)

28. Salo, A., Keisler, J., Morton, A.: Portfolio Decision Analysis. Springer-Verlag New York Inc. (Jul 2011) 